\title{
Role of Isocitrate Dehydrogenase 2, Microphthalmia-Associated Transcription Factor and Alpha Melanocyte Stimulating Hormone in Patients with Non-Segmental Generalized Vitiligo
}

\author{
K.M.Hussein ${ }^{1}$, N.E.Sorour ${ }^{1}$, N.F.Alhusseini ${ }^{2}$, A.G.Abdou ${ }^{3}$ and A.y.Habashy ${ }^{1}$
}

${ }^{1}$ Dermatology and Andrology, Dept., Faculty of Medicine, Benha Univ., Benha, Egypt

${ }^{2}$ Medical Biochemistry \& Molecular Biology, Dept., Faculty of Medicine, Benha Univ., Benha, Egypt

${ }^{3}$ Pathology, Dept., Faculty of Medicine, Menoufia Univ., Menoufia, Egypt

Email: Amalyousif@fmed.bu.edu.eg

Abstract

Background: Vitiligo is a complicated, multifaceted condition of the skin. Abnormalities in the surrounding keratinocytes may lead to death of the melanocytes because growth factors are deprived. Ultraviolet band B (NB-UVB) is a successful treatment option particularly in generalised individuals. Objective: The purpose of this research was to assess in lesional skin of vitiligo patients the levels of microphthalmia-associated transcription factor (MITF), alpha melanocyte stimulating hormone and isocitrate dehydrogenase 2 (IDH2) and the influence of NBUVB treatment on them. Methods: 25 patients have been enrolled. They got NBUVB twice weekly for a total of 20 sessions on non-consecutive days. Each patient received skin biopsies from lesional skin before and after treatment. Results: $10 \%$ of patients had great clinical response after treatment; $10 \%$ showed fair response; $40 \%$ showed moderate reactions; $35 \%$ showed poor reactions; and $5 \%$ exhibited advancing illness. Conclusion: NB-UVB is an effective means of treating vitiligo, by upregulating $\alpha \mathrm{MSH}$, the master regulator of melanogenesis.

Keywords: Vitiligo, NB-UVB, a MSH, Melanogenesis.

\section{Introduction}

Vitiligo is a chronic autoimmune illness that causes white patches on the skin influenced by the loss of melanocytes. Vitiligo affects over $1 \%$ of individuals globally, affecting adults and children alike, causing decreased quality of life and considerable psychological anguish. [3]

While the processes involved in the aetiology of vitiligo are not completely known, oxidative stress might play an important role. Indeed, some mediators, like hydrogen peroxide, are hazardous to melanocytes and are created in a biosynthesis of melanin. If the antioxidant system is not removed, these mediators may damage melanocytes. [4]

The relationship between melanocytes and keratinocytes is closely related to melanogenesis. Melanogenesis is controlled by secretionary substances from surrounding keratinocytes in melanocytes. Keratinocytes generate and release substances such as $\alpha$ melanocyte stimulant $(\alpha-\mathrm{MSH})$ that control and accelerate melanocyte epidermal melanogenesis [17]. $\alpha$ MSH interacts to encourage melanin formation and further specialised melanocyte activities with an unique cell surface receptor called the Melanocortin 1 Receptor (MC1R) [14].

After MC1R is stimulated by its endogenous agonist $\alpha$-MSH, MC1R activates adenylyl cyclase, which leads to an increased intracellular cyclic AMP content (cAMP). CAMP then stimulates the kinase protein A (PKA), which in turn activates the transcription factor family members of CREB cAMP responsive-elementbinding protein (CREB) [15]. CREB enhances the production of many pigmentation-enzymes, including as tyrosinase (TyR), tyrosinase-related protectin 1 (TRP-1), cyrosinase-related protein 2, and melanocytic differentiation markers, through transcriptional activation of a range of downstream targets, including
Microphthalmia-associated transcription factor (MITF). [8]

MITF is a master melanogenesis regulator that upregulates TYR, TRP-1 and TRP-2 melanogenesis enzymes. MITF also controls melanocyte function including differentiation of the melanocyte, pigmentation, proliferation and cell survival. [13]

Many studies have shown that mitochondria plays a role in processes such as melanogenesis. Mitochondria have been demonstrated to influence melanosome biogenesis. [4]

Mitochondria is a major generator of ROS in the skin and plays a crucial role in modifying cellular redoxbased activities such as metabolism or apoptosis. Accumulated reactive oxygen (ROS) species affects both keratinocytes and melanocytes by inducing DNA damage that ultimately leads to stimulation of synthesis of $\alpha$-MSH. [11]

Mitochondrial dehydrogenase 2 (IDH2) is a subtype of dehydrogenase isocitrate and is known to create NADPH in mitochondrines [4]. In converting NADP+ to NADPH IDH2 fulfils the function of antioxidative protein against ROS that helps regenerate reduced glutathione from oxidised glutathione. IDH2 may thus play a major role in melanogenesis [11].

To yet, no therapy for vitiligo has been totally successful. Techniques have been employed to promote repigmentation, such the ultraviolet $\mathrm{B}$ light (NB-UVB) narrow band, now regarded as the preferred therapy for vitiligo. This treatment utilises a $311 \mathrm{~nm}$ light-spectrum that shows penetration in the epidermis' baseline, encourages melanocyte proliferation in the skin and on the external epithelial root sheath of the hair follicle, increases the melanogenesis and the generation of MSH [14]. 


\section{Patients and methods}

\subsection{Subjects}

Twenty five generalised nonsegmental vitiligo patients during the period March 2018 to March 2021, they were chosen from the outpatient clinic of Dermatology, Venerology \& Andrology, Benha University Hospitals. There were 7 men and 18 females aged 18 to 65 with an average of $31.7 \pm 18.9$ years.

The study was authorised by the Institute Review Board (IRB) of Benha University, Faculty of Medicine. All subjects acquired written informed consent.

\subsection{Inclusion criteria}

Generalized non-segmental vitiligo involving $\geq 5 \%$ of body surface area (BSA)

\subsection{Exclusion criteria}

1. Patients received topical therapy within the previous month.

2. Patients received systemic therapy within the previous 3 months.

3. Patients with history of skin cancer or Photosensitivity.

Complete history was taken and detailed dermatological examination was done for all

\subsection{Patients}

\section{Assessment of disease severity}

The Vitiligo Area Scoring Index (VASI) was used. First, the body was separated into five areas: hands, upper limbs (including axils), trunk, feet and lower limbs (including buttocks and inguinal areas). The degree of participation of the hand unit was assessed in each location. One unit, encompassing all digits' palm and palmar surface, accounts for around $1 \%$ of the total BSA. The remaining depigmentation was then represented by the following percentages: 0,10 percent, 25 percent, $50 \%, 75$ percent, $90 \%$ or 100 percent. Only specks have been seen with depigmentation at $10 \%$; at $25 \%$, the depigmented area was less than that of the pigmented area; at $50 \%$, the depigmented and pigmented area was equal; at $75 \%$, the depigmented zone was higher than that of pigmented area; at $90 \%$, pigment particles were left; No pigment remained after $100 \%$ depigmentation.

The VASI has been developed by multiplying the number of hand units by the residual depigmentation per area by the total of these values for all body areas in the following formula:

VASI $=$ [Man Units] [Residual Depigmentation]. Terms of Reference.

(Range possible; 0-100)

UVB Phototherapy Narrow-Band:

All patients got 2 months non-consecutive NB-UVB phototherapy twice a week. This was done using the GH8ST phototherapy equipment.

The gadget includes 8 mercury lamps with a spectrum of $305-315 \mathrm{~nm}$, wavelength of $311 \mathrm{~nm}$ and a brightness of $6-8 \mathrm{~mW} / \mathrm{cm} 2$ (Philips TL 100W/01). NBUVB phototherapy doses were given according to readily calibrated tables provided by the manufacturer.
Clinical Assessment: Before and after each session, patients were evaluated to ascertain their clinical response. Two independent observers examined lesion photographs before and after treatment. The clinical response of patients was rated according to the percentage of repigmentation7.

Excellent response: if repigmentation of depigmented lesions was $>75 \%$ at the end of therapy.

Good response: if repigmentation ranged from 51-75\%. Moderate response: if repigmentation ranged from 26 $50 \%$.

Poor response: if repigmentation ranged from 1-25\%.

Absent response: if no repigmentation was noted.

Worse response: if progressive depigmentation of lesions was noted. Improvement in VASI score after therapy was graded as follows.

Very much improved: if VASI score decreased by more than $50 \%$ at the end of

therapy.

Much improved: if VASI score decreased by 25-50\%.

Improved: if VASI score decreased by $10-25 \%$.

Minimally improved: if VASI score decreased by less than $10 \%$.

No change: if VASI score decreased by $0 \%$.

Worse: if VASI score increased indicating progression of disease.

\section{Skin biopsy}

Two $5 \mathrm{~mm}$ punch biopsies were obtained from each vitiligo patient; from lesional skin. Two biopsies were obtained from the same site after the end of NB-UVB phototherapy. Site of biopsy was sterilized using alcohol then local anaesthesia (Mepecaine ${ }^{\circledR}$ 3\%) was injected subcutaneously. All specimens were fixed in $10 \%$ natural buffered formalin solution. Fixed specimens were then dehydrated in ascending grades of alcohol, cleared in xylol and processed into paraffin blocks. Serial 4 micron thick sections were obtained from each block and stained with

Haematoxylin and Eosin (H\&E) stain for histological diagnosis.

\section{Statistical Analysis}

For data analysis the statistical package SPSS (Statistical Package for the Social Science; SPSS Inc., Chicago, IL, USA) version 22 was used. Data was summarized using mean (X), standard deviation (SD), minimum and maximum in quantitative data and using frequency (count; n) and relative frequency (percentage; \%) for categorical data. The following tests were used; chisquare test (x2), student t-test (t), and paired samples ttest. Correlations were done using Spearman correlation coefficient (r). In all tests, statistical significance was set at $\mathrm{P} \leq 0.05$.

\section{Results}

\subsection{Patient data}

In this study, 25 patients with stable vitiligo were included, 18 women (72\%) and 7 men (28\%) with greater than $10 \%$ of their body surface affected by the disease, and skin photo types III-IV (Fitzpatrick classification. 
Analysis of the disease among the patients was performed regarding the consanguinity, family history of the disease, associated autoimmune diseases, duration, exacerbating factors for the disease, activity of the disease, other associated systemic diseases together with intake of drugs for these diseases, greying of hairs and skin phenotype.

In this study, out of 25 patients, only 4 patients (18\%) reported positive consanguinity. Also 7 patients (30\%) had a similar condition in their family.

During taking the medical history, no history of any associated auto immune diseases among the studied patients. Only $2(8 \%)$ patients had diabetes mellitus, 6 (12\%) patients were hypertensive and $1(4 \%)$ patients reported intake of systemic drugs for their diseases.

As regarding to the duration of the disease, 14 $(58 \%)$ patients reported that they had vitiligo for less than 10 years, while the rest $(42 \%)$ suffered from the disease for 10 years or more.

By studying the disease stability among studied patients, $9(38 \%)$ patients reported new lesions in less than 3 months, while the rest of the patients (62\%) reported stable disease for more than 3 months.

There were several exacerbating factors for the disease among studied patients. 10 (40\%) patients had only one exacerbating factor for their disease, 10 (40\%) patients had several exacerbating factors while the rest (20\%) did not report any factor. Stress was the commonest factor could exacerbate the disease in our studied patients $(76 \%)$, trauma $(28 \%)$, sun exposure $(16 \%)$ and lastly infections $(4 \%)$.

The degree of clinical improvement was assessed objectively. The objective response was evaluated by calculation of VASI score and VETI score. The extent of improvement was detected by subtraction of VASI score calculated at start of treatment from that calculated at 2nd month after treatment then dividing the result on recorded VASI at 2nd month. Also the same is done for VETI score.

By comparing the degree of improvement by VASI at 0 and 2nd month after treatment, it was found that both are significantly related to each other.

The degree of improvement among studied patients was assessed, it was found that all patients were improved but with different degrees. 1 patients was minimally improved, 12 patients were improved, 9 patients were much improved and 3 patients showed very much degree of improvement.

Photographs were used to document the extent and severity as well as to monitor the degree of improvement. Samples of the studied patients before and after treatment are illustrated in

Before treatment, histopathological examination of H\&E stained sections revealed

absent melanin pigment and melanocytes in lesional skin in all 25 patients $(100 \%)$. After NB-UVB phototherapy, there were variable degrees of partial restoration of melanocytes and melanin pigment in lesional skin in 48 patients $(95 \%)$ with 1 patient showing evident dermal melanophages. Persistent absence of melanin pigment and melanocytes was noted in lesional skin in only two patienst. (8\%) who showed progressive disease.

Table (1) Age and sex distribution among studied groups.

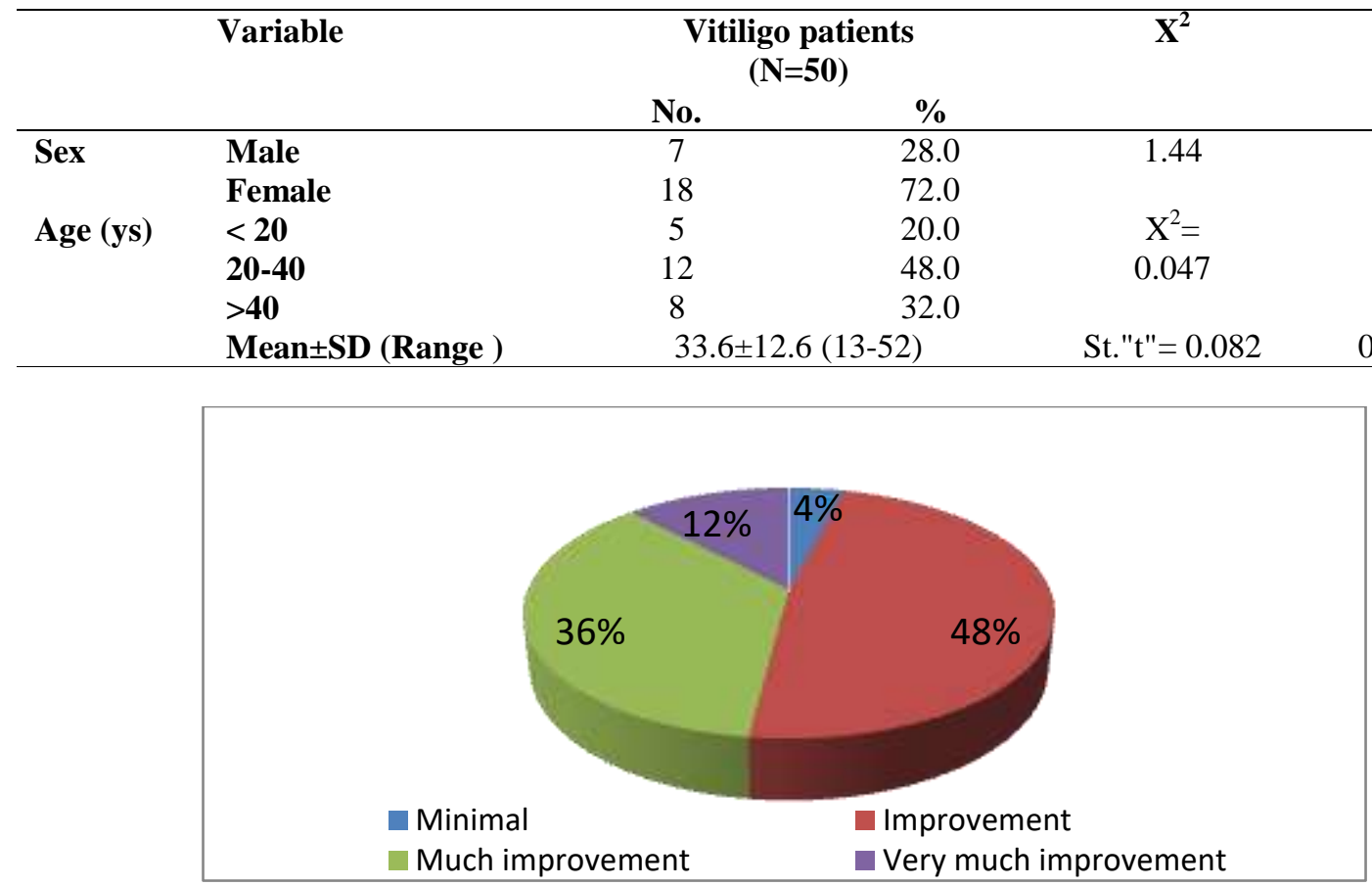

Fig. (1) The degree of clinical improvement. 


\section{Discussion}

This study included 25 patients with generalized non-segmental vitiligo including 7 male patients $(28 \%)$ and 18 female patients $(72 \%)$ with the male to female ratio being

2.33 to 1 . This is consistent with previous studies that reported development of vitiligo predominantly in male individuals [23]. In contrast, other studies reported predominant affection of females [20].

$\mathrm{Nb}-\mathrm{UVB}$ phototherapy has been considered the first choice treatment in vitiligo vulgaris as it is effective and well-tolerated [19]. In this study, all patients treated for degree of repigmentation, reaffirming the usefulness of nb-UVB phototherapy as a therapeutic alternative in cases of stable vitiligo. The UV radiation can upregulate $\alpha$-MSH level and activity, POMC expression, and POMC peptide production; include $\alpha$-MSH, $\beta$-endorphin, and ACTH molecules that participate in the regulation of skin pigmentation, to protect skin from UV induced injury, and to modulate skin immune response [20]. It's well known that UV light stimulates the production of endothelin-1 (ET-1) and POMC by keratinocytes, factors that can act in a paracrine manner by stimulating the function of melanocytes. [20]

$\mathrm{Nb}-\mathrm{UVB}$ phototherapy acts through the induction of local immunosuppression and the stimulation of the proliferation of melanocytes in the skin and in the outer sheath of the hair follicle root; there is also a stimulating effect on melanogenesis and on the production of melanocyte-stimulating hormone (MSH). [18]

Skin pigmentation is due to the pigment produced by melanocytes, eumelanin and pheomelanin, and their accumulation on melanin granules in the keratinocytes. These pigments produced by melanocytes are responsible for skin and hair color. Skin pigmentation is an important protective mechanism against the DNA damaging and mutagenic effects of solar UV radiation. [21]

The melanocortin system can play an important role in the pathogenesis of vitiligo, and can be affected by this disease, as was reported in the studies of Kingo. [18]

In conclusion, $\mathrm{Nb}-\mathrm{UVB}$ phototherapy is effective in the treatment of patients with stable vitiligo, favouring repigmentation, through upregulation of $\alpha-\mathrm{MSH}$. Although we reaffirm that the melanocortin system intervenes in the pathogenesis of stable vitiligo.

Fundin

The datasets used and/or analyzed during the present study are available from the corresponding author on reasonable request.

\section{Authors' contributions}

All authors approved the final version of the manuscript.

\section{Ethics approval and consent to participate}

The University Hospital-UANL Institutional Review Board approved and registered the study.

\section{Patient consent for publication}

All patients consented to the publication of their data and associated images.

Competing interests
The authors declare that they have no competing interesets.

\section{References}

[1] KM. Alghamdi, A.Kumar, A.Taieb and K. Ezzedine Assessment methods for the evaluation of vitiligo. J Europ Acad Dermatol Venereol.vol.26,pp.1463-1471,2012.

[2] A.Bhatnagar, A J.Kanwar, D.Parsad and D.De. Psoralen and ultraviolet $\mathrm{A}$ and narrow band ultraviolet $\mathrm{B}$ in inducing stability in vitiligo, assessed by vitiligo disease activity score: an open prospective comparative study. J Eur Acad Dermatol Venereol.vol.21,pp. 1381-1385. ,2007.

[3] K.Bonotis, K.Pantelis and S.Karaoulanis. Investigation of factors associated with healthrelated quality of life and psychological distress in vitiligo. J Dtsch Dermatol Ges.vol.14,pp.4549,2016.

[4] T.Daniele, I.Hurbain, R.Vago, G.Casari, G.Raposo, C.Tacchetti . Mitochondria and melanosomes establish physical contacts modulated by Mfn2 and involved in organelle biogenesis. Curr Biol.vol.24(4),pp.393-403,2014.

[5] K.Ezzedine, HW.Lim, T.Suzuki, I.Katayama, I.Hamzavi, CC.Lan. Revised classification/nomenclature of vitiligo and related issues: the Vitiligo Global Issues Consensus Conference. Pigment Cell Melanoma Res.vol.25,pp.1-13,2012.

[6] A.Feily. Vitiligo Extent Tensity Index (VETI) score: a new definition, assessment and treatment evaluation criteria in vitiligo. Dermatol Pract Concept.vol. 4(4),pp.81-84,2014.

[7] JC.García-Borrón, Z.Abdel-Malek and C.JiménezCervantes. MC1R, the cAMP pathway, and the response to solar UV: extending the horizon beyond pigmentation Pigment cell\& melanoma research.vol. 27(5),pp.699-720,2014.

[8] NB.Goldstein, MI.Koster, LG. Hoaglin, NS. Spoelstra, KJ. Kechris, SE. Robinson . Narrow Band Ultraviolet B Treatment for Human Vitiligo Is Associated with Proliferation, Migration, and Differentiation of Melanocyte Precursors. Journal of Investigative Dermatology.vol. 135,pp. 20682076,2015.

[9] I.Hamzavi, H.Jain, D.McLean, J.Shapiro, H.Zeng and H.Lui. Parametric modeling of narrow band UVB phototherapy for vitiligo, using a novel quantitative tool: the vitiligo area scoring index. Arch Dermatol.vol.140,pp.677-683,2004.

[10] H.W.Lim, P.E.Grimes, O.Agbai, I.Hamzavi, M.Henderson, M.Haddican. Afamelanotide and Narrowband UV-B Phototherapy for the Treatment of Vitiligo: A Randomized Multicenter Trial JAMA Dermatol.vol.151(1),pp.42-50,2015.

[11] D.E.Lin JYand Fisher. ( Melanocyte biology and skin pigmentation Nature.vol.445,pp.843-850, 2007. 
[12] M.Mirnezami and H.Rahimi, Serum Zinc Level in Vitiligo: A Case-control Study. Indian J Dermatol.vol.63(3),pp. 227-230,2018.

[13] C.Niu, L.Yin and HA.Aisa. Novel Furocoumarin Derivatives Stimulate Melanogenesis in B16 Melanoma Cells by Up-Regulation of MITF and TYR Family via Akt/GSK3 $/ / \beta-C a t e n i n$ Signaling Pathways Int. J. Mol. Sci.vol. 19(3),pp. 746-765,2018.

[14] J.Ocampo-Candiani, M. Salinas-Santander, V. Trevino, R. Ortiz-López, Ocampo-Garza J and CN. Sanchez-Dominguez. Evaluation of skin expression profiles of patients with vitiligo treated with narrow-band UVB therapy by targeted RNAseq. An Bras Dermatol.vol.93(6),pp.843-851,2018.

[15] JH. Park, HJ. Ku, JH. Lee and JW. Park. IDH2 deficiency accelerates skin pigmentation in mice via enhancing melanogenesis. Redox Biology.vol. 17,pp.16-24,2018.

[16] M.Salinas-Santander, V.Trevino, E. De la RosaMoreno, B.Verduzco-Garza, CN. SánchezDomínguez, C.Cantú-Salinas . CAPN3, DCT, MLANA and TYRP1 are overexpressed in skin of vitiligo vulgaris Mexican patients. Exp Ther Med.vol.15(3),pp.2804-2811,2018.

[17] YH .Shin, YK. Seo, HH. Yoon, KY .Song and JK. Park. Effect of keratinocytes on regulation of melanogenesis in culture of melanocytes, Biotechnol. Bioprocess. Eng.vol.17,pp.203210,2012.

[18] K.Kingo, E.Aunin, M. Karelson. MA.Philips, R.Rätsep. Gene expression analysis of melanocortin system in vitiligo. J Dermatol Sci.vol. 48,pp.113122. doi: 10.1016/j.jdermsci.2007.06.004. Majid I. Vitiligo management: An update. BJMP, 2007.

[19] G.Stinco, G.Trevisan, C.Buligan, G.Gregoraci, S.De Marchi, N.di Meo, P.Patrone Narrow bandultraviolet $\mathrm{B}$ versus clobetasol propionate foam in the treatment of vitiligo: A retrospective study. Dermatol Ther (Heidelb) .vol.3,pp.95-105, 2013.

[20] AT. Slominski, MA. Zmijewski, PM. Plonka, JP. Szaflarski, R. Paus. How UV light touches the brain and endocrine system through skin, and why. Endocrinology. 2018;159:1992-2007. doi: 10.1210/en.2017-03230. Costin GE, Hearing VJ. Human skin pigmentation: Melanocytes modulate skin color in response to stress. FASEB J.vol.21,pp.976-994. doi: 10.1096/fj.066649rev,2007.

[21] VB. Swope, ZA. Abdel-Malek. MC1R: Front and center in the bright side of dark eumelanin and DNA repair. Int J Mol Sci.vol. 19(pii),pp.E2667,2018.

[22] NA.Swope, SB. Mahmoud, RM. Abdel Hay, MM .Hassieb, LA. Rashed. Assessment of gene expression levels of proopiomelanocortin (POMC) and melanocortin-1 receptor (MC1R) in vitiligo. Australas J Dermatol.vol.58,p.e36-e39. doi: 10.1111/ajd.12408,2017.
[23] DS.Shankar, K. hashikala, R.Madala. Clinical patterns of vitiligo and its associated co morbidities: a prospective controlled crosssectional study in South India. Indian Dermatol Online J.vol.3 (2),pp.114-118,2012.

[24] A. Lahlou, H. Baybay, S .Gallouj, FZ. Mernissi. Childhood vitiligo: Clinical epidemiological profile. Our Dermatol Online.vol.8(3),pp.264-267, 2017. 